\title{
Choosing the right place? \\ An experimental examination of ethnic preferences in housing decisions
}

\author{
Christoph Zangger*
}

\begin{abstract}
Residential preferences are often treated as exogenous causes of social and ethnic segregation. In this paper, this assumption is challenged. Instead, this contribution proposes and evaluates how residential preferences are shaped by the conditions of one's neighbourhood. This approach acknowledges the mutual dependence of experienced neighbourhood and housing conditions, residential preferences, and segregation patterns. Doing so, an alternative explanation for the often documented ethnic preferences in housing decisions is elaborated and tested by applying multilevel generalized linear latent and mixed logit models to unique, geocoded data from a choice experiment. Results indicate that people differ in their evaluation of the social and ethnic composition of housing alternatives' residential surroundings. Moreover, these heterogeneous evaluations are largely independent of respondents' own ethnic background. Instead, the observed taste heterogeneity varies over respondents' bespoke neighbourhoods. However, the evaluation of the social and ethnic composition of the housing alternatives does not vary over larger administrative neighbourhoods. This result highlights the importance of proximate, small-scale geographic processes in shaping people's residential preferences.
\end{abstract}

Keywords: Housing Choice, Residential Preferences, Choice Experiment, Ethnic Background

\section{Introduction}

Ethnic and social segregation and its consequences for individual life chances are an enduring hot topic on both the political as well as researchers' agenda (e.g., Iceland and Hernandez, 2017; Rokem and Vaughan, 2019; Sampson, 2012). Following Schelling's (1971) well-known model of (ethnic)

\footnotetext{
*University of Zurich, zangger@soziologie.uzh.ch
} 
tipping points in people's residential mobility decisions, researchers have intensively studied the formation of residential preferences and their consequences in terms of social and ethnic segregation (e.g., Aldén et al., 2015; Clark, 1991; Emerson et al., 2001). However, residential preferences only explain part of the observed levels of segregation in today's metropolitan areas. People's situational context, in particular the resources they possess, also crucially determines their residential mobility and housing choices (e.g., Iceland and Hernandez, 2017; Musterd, 2005). Moreover, ethnic residential segregation can also be attributed to discriminatory practices of landlords who limit the scope of choice for minorities (e.g., Auspurg et al., 2017; Flage, 2018). These complex and intertwined causes make it hard to isolate the contribution of people's preferences for patterns of residential segregation.

When it comes to assessing people's residential preferences, most studies use either the observed housing decisions or simulation studies (e.g., Clark and Fossett, 2008; Emerson et al., 2001; Fossett, 2006; van Ham and Clark, 2009). With the former, however, it is not possible to fully disentangle people's preferences from unobserved characteristics, tastes, opportunities, and limitations that are associated both with their observed choice and the predictors used in the model. Meanwhile, only few studies have tried to study residential preferences directly by means of survey experiments (e.g., Bruch and Mare, 2006; Ibraimovic and Hess, 2018). This is surprising given that these methods bear the advantage of causally revealing the factors that shape the formation of people's preferences (Train, 2009). Moreover, focusing on preferences rather than choices is also valuable because preferences can be understood as people's demand for housing which can be used for future planing and development.

Against the background of the documented neighbourhood influences on people's residential mobility decisions (Lee et al., 1994), this contribution examines how people's residential preferences are formed by the conditions they experience in their neighbourhoods. More specifically, using a choice experiment in which the social and ethnic composition of different housing alternatives was systematically varied paired with geocoded data that allows for the construction of bespoke neighbourhoods, this contribution evaluates how people's residential preferences differ across neighbourhoods. Doing so, this contribution also examines how experienced neighbourhood conditions add to the explanation of varying responses to the social and ethnic composition of available housing alternatives. Consequently, preferences are not treated as an exogenous cause of residential segregation. Instead, this paper stresses the importance of people's experienced neighbourhood context to explain varying residential preferences. To this end, experienced neighbourhood conditions, individual background, and characteristics of potential housing alternatives are incorporated and tested in a coherent model. 
The remainder of this contribution is organized as follows. The next section briefly summarizes existing research and elaborates on the theoretical background. The third section discusses the data and the methodological approach used to assess the research question, namely the choice experiment, the construction of bespoke neighbourhoods, and the pursued mixed logit modelling strategy (Train, 2009). The results of different model specifications are presented in the fourth section. Finally, the fifth section concludes with a critical discussion of the results and summarizes the implications for both research and policy concerned with residential mobility and social and ethnic segregation.

\section{Background}

To evaluate people's residential preferences, housing decisions are framed in a broad rational action model. Taking different constraints and opportunities into account, for example, the availability of different housing alternatives on the market or individual monetary resources, people prefer the housing alternative that is associated with the highest subjective utility, for example, with regard to housing characteristics or life cycle events (e.g., Ioannides, 1987; McFadden, 1978; Rossi and Shlay, 1982):

$$
\max U_{j}=U\left(\mathbf{Z}_{j}\right)
$$

That is, people try to maximize the utility associated with different observed and unobserved factors $\mathbf{Z}_{j}$ over housing alternatives $j$. Given the subjective evaluation of different housing alternatives, it has been suggested that the observed levels of social and ethnic segregation are also partly due to varying preferences of different social and ethnic groups (e.g., Clark, 1991; Schelling, 1971). In the present context, this implies that some groups might emphasise certain commodities or the composition of a neighbourhood $j$ when comparing it to an alternative $k$ while others would largely be indifferent to, for example, the social and ethnic background of their prospective neighbours.

There is an ever growing literature focusing on the factors $\mathbf{Z}_{j}$ that shape the utility associated with a particular housing alternative. On the one hand, there is considerable research focusing on the individual and joint decision making processes of households in residential choices, pointing to, for example, differences across social strata, age, change of workplace, or family size (e.g., Dieleman, 2001; Wang and Li, 2006; Weinberg, 1979). On the other hand, there is research that puts residential mobility into the larger context of the available housing alternatives and their respective neighbourhoods (e.g., Clark and Fossett, 2008; Lee et al., 1994; Timmermans and Noortwijk, 1995; van Ham and Clark, 2009).

Although it can be considered to be the foundation of the well-known model of segregation by 
Schelling (1971), bringing the individual and the contextual perspective on people's residential preferences together has often proven to be problematic. In this regard, explanations that focus on individual preferences in the housing market often neglect structural constraints and discrimination experienced by ethnic minorities as well as the fact that preferences themselves are also the result of experienced neighbourhood conditions (Bruch and Feinberg, 2017; Bruch and Mare, 2006). This last point is then also the motivation for the present research: Preferences are not fully individual but they are dynamic and formed in a social environment (Ioannides, 2013; Lévy-Garboua et al., 2006). Regarding residential preferences, we would thus expect that people update their evaluation of what the ideal neighbourhood looks like based on the experiences made in their current (and previous) surroundings. This in turn then also influences their evaluation of different housing alternatives.

Against the background of the formation of individual residential preferences in a socio-spatial context (Bruch and Feinberg, 2017; Ioannides, 2013), this paper hypothesizes that ethnic preferences in housing decisions are also attributable to people's perception of the social environment of their current neighbourhood. In other words: Rather than a true cause of observed levels of segregation, the often documented ethnic differential in people's location and housing decisions is also a consequence of segregation patterns itself and the resulting social and ethnic composition of people's residential surroundings. This view then stresses a mutual dependence of segregation, residential mobility, and housing preferences (Bruch and Mare, 2012). With regard to the above outlined utility maximization in people's residential decision making, we would thus expect that currently experienced neighbourhood conditions are important determinants when evaluating different housing alternatives and thus crucially shape the utility attributed to different housing opportunities and the therewith associated neighbourhood conditions.

\section{Data \& methods}

This contribution uses a choice experiment and geocoded data collected in four major cities in Switzerland (Basel, Bern, Winterthur, and Zurich). Since discrete choice experiments allow for the identification of causal factors in the formation of people's preferences, they are especially suited for this purpose (Johnson et al., 2013; Train, 2009). Before discussing the design and data in detail, the next section briefly outlines the general idea of discrete choice experiments, their construction, benefits, and limitations. 


\section{Discrete choice experiments}

Discrete choice experiments (DCE) combine - as any type of factorial survey experiment (Auspurg and Hinz, 2015; Zangger and Becker, 2019) - the advantages of traditional experiments with the ones of classic survey research. Unlike in the case of revealed preferences, that is, people's observed choices in the real world, they are used to assess stated preferences, that is, choices in a hypothetical situation (Train, 2009).

Making use of a random assignment of respondents to treatment conditions and an efficient manipulation of different treatment combinations, DCEs allow for the identification of causal effects. While real-world choices are often conducted in a narrow context with only little variation in the attributes of interest (e.g., people's responsiveness to house prices and rents might be underestimated when housing is in short supply), DCEs try to mimic a situation free from such (unobserved) constraints. Since respondents are based on a random sample from the population of interest rather than a (self-) selected group of participants as in laboratory experiments, the high internal validity of experimental designs is paired with the external validity of survey research methods (Zangger and Becker, 2019). Consequently, they make a generalization of respondents' preference structure to the population of interest more feasible.

In a typical DCE, respondents are confronted with a choice set of at least two different alternatives. The alternatives consist of a combination of different levels of attributes that are expected to influence people's choice. Since the set of all potential combinations (commonly referred to as full factorial) is usually too large for practical purposes, researchers seek to reduce the number of different choice sets by means of fractional factorial designs that ensure both the orthogonality of the different dimensions and level balance. Consequently, the manipulated dimensions are independent of each other and each level appears, on average, about the same number of times, ensuring a maximum level of precision and unbiased estimates (Auspurg and Hinz, 2015). Doing so, an efficient identification of causal effects is still possible although not all treatment combinations are presented to the respondents.

However, DCEs are not free of criticism. While they might be adequate to reveal respondents preferences, such preferences do not automatically correspond to choices in the real world (Train, 2009). Likewise, the dimensions in a DCE must contain all the necessary information for respondents to make their decision in the hypothetical situation. If crucial information is lacking, respondents might resort to unobserved heuristics that - in the best case - introduce additional noise and thus lower the statistical precision of estimates or even introduce bias if such heuristics are systematically associated with one or several of the dimensions in the DCE (Johnson et al., 
2013). It is thus important that all the necessary context to make a decision is provided in a DCE. At the same time, the complexity of the hypothetical situation should be limited and the number of experimentally altered dimensions should not be too large. ${ }^{1}$

\section{Data}

In the present case, each respondent was presented one choice set with three alternatives. A sample choice set is depicted in Figure A1 in the Appendix. In the DCE, respondents were asked to help a friend who needs a new place to stay. All three alternatives were described as suiting their friend and it was thus up to the respondents to make a recommendation. Additional contextual information about the situation of respondent's hypothetical friend was provided in a short introduction at the beginning of the DCE. It included her or his household's composition and income. All the experimentally altered dimensions of the DCE and the situational context are summarized in Table 1.

[Insert Table 1 here]

Based on existing literature and a quantitative pretest, seven dimensions were manipulated in the experimental design: the size of the apartment or house (in square meters), the type of housing (a house, an early $20^{\text {th }}$ century apartment, and an apartment in a housing block), the distance to the city centre (in minutes), the rent, an indicator for the neighbourhood's social and ethnic composition (measured as the type of local stores), whether there are parks or other recreational amenities nearby, and the physical state of the neighbourhood environment (whether streets, sidewalks, and gardens are well-kept, have graffiti, or even garbage). Moreover, the number of rooms for each of the alternatives and that the dwelling had been renovated in the course of the last five years was included as additional but constant information.

Given that the full factorial for this generic DCE was rather large (amounting to $3^{6} \times 2=1^{\prime} 458$ treatment combinations), a fractional factorial $D$-efficient design of 162 choice sets was constructed. The relative $D$-efficiency of the final design was 76.94. This design allows for the identification of the main effects of all dimensions of interests as well as interactions of the rent dimension with the size dimension and interactions of the indicator of a neighbourhood's social and ethnic composition with the physical state of a neighbourhood's environment.

The choice experiment was included in a survey on people's living conditions in four Swiss cities. Respondents were chosen through a stratified cluster random sampling. In a first step, administrative neighbourhoods in the cities of Basel, Bern, Winterthur, and Zurich were chosen at random, using the size of inhabitants in each as a probability weight. This resulted in 77 administrative 
neighbourhoods to be included in the survey. These administrative neighbourhoods vary in size (from roughly 2'000 up to 30'000 inhabitants) and comprise, on average, 11' 855 inhabitants. At the same time, these administrative neighbourhoods have their own history and are well known to inhabitants. In a second step, a total of 100 coordinates within these administrative neighbourhoods were drawn at random. These random coordinates are the centres around which bespoke neighbourhoods were constructed. Finally, the 20 households closest to this random coordinates were invited to participate in the survey. If the coordinates corresponded to a housing block with more than 20 households, the latter were selected at random within that particular housing block. Given this sampling strategy, the data allows for the construction of geocoded bespoke neighbourhoods of door-to-door neighbours, nested in larger administrative neighbourhoods in the four cities.

To complete the survey, respondents could either use a paper and pencil or online version of the questionnaire. The response rate for this mixed-mode survey was about $42 \%$, resulting in 823 data sets. After deleting observations with missing values on the dependent variable and those with less than 3 neighbours in bespoke neighbourhoods, this number reduces to 798 observations. Although the participation was voluntary and no incentives were used, this response rate is - at least for the Swiss context-rather high. Nevertheless, non-response is likely selective. This is especially relevant when examining the result of the choice experiment for different subgroups. Consequently, a straightforward generalization of the results is not feasible. However, the combination of an experimental design with geocoded survey data nevertheless depicts a unique opportunity to study contextual influences in the formation of residential preferences.

Both the administrative and the bespoke neighbourhoods vary considerably regarding their ethnic composition. The share of people without Swiss citizenship varies between $16.8 \%$ and $54.9 \%$ in administrative and between $0 \%$ and $85.71 \%$ in bespoke neighbourhoods. Of the respondents in the sample, $32 \%$ were not born in Switzerland and $26.9 \%$ do not have Swiss citizenship. Descriptive statistics of all the measures used in the analysis are available in Table A1 in the appendix.

\section{Methodological approach}

Given the setup of the experiment, a choice modelling approach is followed to investigate people's residential preferences. Assuming that the observed choice in the choice experiment depicts the alternative $j$ to which respondent $i$ attributes the highest utility, a natural model to consider is McFadden's (1974) conditional logit model. This approach models the probability that alternative 
$j$ is chosen by respondent $i$ as

$$
\operatorname{Pr}(Y=j \mid \mathbf{Z})=\frac{e^{\mathbf{Z}_{\mathbf{j}} \boldsymbol{\beta}}}{\sum_{k=1}^{K} e^{\mathbf{Z}_{k} \boldsymbol{\beta}}} .
$$

However, such a model only allows for effects of characteristics that differ between alternatives. While this is no problem for the analysis of the dimensions in the choice experiment, the characteristics of the respondent are constant across alternatives. Likewise, since the DCE in the present case is a generic rather than a branded choice experiment (Kuhfeld, 2010), respondent main effects do not have any substantial meaning. ${ }^{2}$ To assess whether people born abroad differ in their residential preferences from those born in Switzerland, one thus needs to either include interaction terms of individual background characteristics and the dimensions of interest or run the analyses separately for migrants and people born in Switzerland.

Conditional logit models, however, rely on the assumption of an independence of irrelevant alternatives (IAA - Train, 2009): For any two alternatives $j$ and $k$, the ratio of the choice probabilities only depends on the dimensions of these alternatives (the common denominator of both choice probabilities from equation (2) cancels out). Given the generic design of the present choice experiment, this assumption is rather unrealistic. A framework that allows for unrestricted substitution patterns and random taste variations are mixed logit models. Mixed logit models express the choice probabilities in the form

$$
\operatorname{Pr}(Y=j)=\int L_{j}(\boldsymbol{\beta}) f(\boldsymbol{\beta}) d \boldsymbol{\beta},
$$

where

$$
L_{j}(\boldsymbol{\beta})=\frac{e^{V_{j}(\boldsymbol{\beta})}}{\sum_{k=1}^{K} e^{V_{k}(\boldsymbol{\beta})}}
$$

is the logit choice probability evaluated at parameters $\boldsymbol{\beta}$ and $V_{j}(\boldsymbol{\beta})$ is the observed portion of the utility that depends on $\boldsymbol{\beta}$ (Train, 2009).

In the present context, we take the conditional logit models as a starting point to estimate the (fixed) influence of the different dimensions in the choice experiment on respondents' residential preferences. In a first step, the analyses are conducted separately for people with and without a migration background in order to test whether there is evidence for an ethnic gradient in people's residential preferences. We then relax the IIA and check for random taste variations in $\boldsymbol{\beta}$ (Train, 2009). More specifically, it is evaluated if such a variation can be attributed to particular groups of respondents, namely whether people with and without a migration background differ in their evaluations of a potential neighbourhood's social and ethnic composition. Finally, the variation 
in $\boldsymbol{\beta}$, that is, respondents' heterogeneous preferences, are linked to their own bespoke and administrative neighbourhoods using multilevel generalized linear latent mixed models (Skrondal and Rabe-Hesketh, 2003). Doing so, it is evaluated to which extent varying preferences might be the result of different experienced neighbourhood conditions.

\section{Results}

\section{Assessing people's residential preferences}

The first two columns in Table 2 depict the effects (log odds) of the experimentally altered dimensions on people's housing preferences when using conditional logit models. Comparing the results for people who were born in Switzerland with those who were born abroad, we generally notice a similar pattern of effects. Both groups prefer larger dwellings. This effect, however, is more pronounced for people with a migration background. Likewise, an increasing distance of a housing option to the city centre is associated with a lower likelihood of choosing that particular alternative: A one minute increase in the distance leads to a $3 \%\left(e^{-0.029}\right)$ decrease in the odds of choosing that alternative. Unsurprisingly, a higher rent decreases the likelihood to choose a particular housing alternative.

There are, however, some more pronounced differences between people with and without a migration background in the evaluation of the type of housing as well as the neighbourhood's social and ethnic composition. While both groups do not differentiate between a single house or an early $20^{\text {th }}$ century apartment (both are in short supply in Swiss cities), people born in Switzerland dislike living in a housing block. On the other hand, people with a migration background do not discriminate between the three different types of housing. Regarding the social and ethnic composition of an available housing alternative, people born in Switzerland prefer to live in a 'typically Swiss' neighbourhood, that is, a neighbourhood which is described as mainly featuring well-known retailers. If the neighbourhood has mainly Turkish and Tamil shops, an indicator for an ethnically diverse neighbourhood, the odds of preferring that alternative are approximately

$60 \%$ lower $\left(e^{-0.933}\right)$. Surprisingly, this effect is even more pronounced for people not born in Switzerland. Their odds of preferring a housing alternative in an ethnically mixed neighbourhood are almost $65 \%\left(e^{-1.029}\right)$ lower. At the same time, people with a migration background also dislike living in a gentrifying, upper class neighbourhood, in which local businesses are mainly cafés and small boutiques. Although the effect is in the same direction, the coefficient for people born in Switzerland is not significant at the conventional 5\%-level.

Similar patterns of effects for people with and without a migration background can be found 
in the case of the remaining experimentally altered dimensions. Both groups prefer having public green spaces such as parks close to the their home. Likewise, the indicator of the physical state of the neighbourhood has similar effects for both groups. If the neighbourhood of a particular housing alternative is described to have graffiti on some walls rather than clean gardens, streets, and sidewalks, the likelihood of choosing that particular neighbourhood is significantly lower. This effect is even more pronounced if the neighbourhood is described to have garbage and bulky waste on sidewalks and streets.

Finally, although not shown in Table 2, the interaction of a neighbourhood's social and ethnic composition with the physical state of the neighbourhood is not significant. Likewise, interacting the indicator of household composition (presence of school-aged children) with a housing alternative's social and ethnic composition as well as the physical state of the neighbourhood does not lead to conclusive results either. Moreover, using the relative rent, that is, dividing the rent by the income of their hypothetical friend leads to the same result as when this contextual information is not included.

[Insert Table 2 here]

As a more straightforward examination of the effects, respondents' willingness to pay (WTP) for each of the categorical dimensions in the choice experiment was calculated (Hole, 2007). The WTP is obtained by dividing the estimated coefficient of interest by the cost coefficient from the conditional logit models in Table 2. More specifically, the WTP for a change in dimension $k$ is given as

$$
W T P_{k}=-\frac{\beta_{k}}{\beta_{C}},
$$

where $\beta_{C}$ is the cost parameter, that is, the estimated effect of the rent to pay for a housing alternative. $^{3}$ Figure 2 depicts the result of this exercise. Both respondents born abroad as well as those born in Switzerland are willing to pay about 400.- Swiss Francs more to avoid living in an ethnically mixed neighbourhood. Likewise, they would only live in a more affluent, gentrifying neighbourhood if the monthly rent was approximately 200.- Swiss Francs less. This effect, however, is only marginally significant for people without a migration background. By far, the biggest effect can be attributed to the state of the residential environment. Especially people who were not born in Switzerland are willing to pay up to 600.- Swiss Francs more to live in a neighbourhood with well-kept gardens and clean streets instead of in a neighbourhood that depicts physical signs of disorder. Compared to the average rent in the choice experiment (amounting to 2119.- Swiss Francs per month), this corresponds to a $28.3 \%$ higher rent to avoid such, presumably, disadvantaged neighbourhoods. 
[Insert Figure 1 here]

So far, we assumed that the evaluation of different attributes and dimensions of the choice experiment is fixed and that respondents show a proportional substitution pattern in line with the IIA (Train, 2009) — at least within the evaluated subgroups of people born in Switzerland and those born abroad. In the next section, we evaluate this assumption using mixed logit models and assess the heterogeneity in people's residential preferences.

\section{Accounting for heterogeneous tastes}

Some respondents might care more about certain attributes than others do (preference heterogeneity). To account for such heterogeneous tastes, mixed logit models with correlated random effects were estimated (Train, 2009). Columns 3 and 4 in Table 2 depict the results of these models. Alternatively, it would also be possible to model the heterogeneity by scaling the idiosyncratic error for each respondent (Louviere et al., 2008). Doing so, however, results in an inferior model fit.

While the pattern of the fixed effects is roughly the same as in the case of the conditional logit models, the mixed logit models reveal significant variation among respondents when evaluating the indicator of a neighbourhood's social and ethnic composition. This is especially the case when the neighbourhood is described as being ethnically diverse. Moreover, the fixed coefficient for a neighbourhood's ethnic composition is now much larger for people born in Switzerland, indicating that they are, on average, more likely to dislike living in ethnically mixed neighbourhoods. The more pronounced heterogeneous tastes when evaluating a neighbourhood's ethnic composition is likely also due to the crude differentiation between native born and first generation migrants: Both groups are quite heterogeneous. While people born in Switzerland are partly second generation migrants, the first generation comprises respondents from neighbouring countries that are very similar to people without any migration background as well as other groups of migrants with a very distinct social and economic background. Grouping people from (north-)western European countries and those from Anglo-Saxon countries together with those without any migration background at all, however, leads to very similar results.

The mixed logit model can be used to save individual-level parameters using a simulation approach (Train, 2009). Figure 2 depicts the density of the individual random parameter for both categories of a neighbourhood's social and ethnic composition. In this regard, it becomes apparent that people differ considerably in their evaluation of a neighbourhood's social and ethnic composition. While the random parameters are roughly centred around the values of the fixed effects 
estimates $(\hat{\beta})$, they depict a considerable spread. Some observations have positive values, indicating that these respondents regard either a gentrifying, more affluent neighbourhood (cafés \& boutiques) or a ethnically mixed neighbourhood (Turkish \& Tamil shops) as a desirable residential environment. While the distributions of people with and without a migration background are almost identical in the case of gentrifying neighbourhoods, they differ somewhat in their response to an ethnically mixed neighbourhoods. The probability mass of the random effect's distribution of people born abroad is to the right of the one of people born in Switzerland. Although the vast amount of the distribution is still below zero, this indicates that people born abroad generally are less sceptical about living in ethnically mixed neighbourhoods than their native-born counterparts.

[Insert Figure 2 here]

In the remainder of this section it is evaluated to what degree this heterogeneous responses to a neighbourhood's social and ethnic composition can be explained by respondents' own neighbourhoods. To this end, multilevel generalized linear latent mixed models (GLLAMM - Skrondal and Rabe-Hesketh, 2003) were estimated.

\section{Evaluating the link to people's own neighbourhood}

The last two columns in Table 2 depict the result of allowing for respondents' evaluation of a neighbourhood's social and ethnic composition to vary as a function of their own administrative and bespoke neighbourhood. However, given the small number of respondents with a migration background and the complexity of such models, running separate models for people born in Switzerland and those born abroad is not feasible. Instead, the model is estimated for the whole sample. ${ }^{4}$ While the fixed parameter estimates are again in line with the effects found in the previous models, the explanatory power of respondents' own neighbourhood composition varies with the scale at which such neighbourhoods are constructed.

On the one hand, the second-last model in Table 2 informs us that the evaluation of a housing alternative's social and ethnic surroundings does not vary over people's current administrative neighbourhoods. On the other hand, allowing for response heterogeneity at the level of bespoke neighbourhoods seems to be more promising. In the last model of Table 2, there is a significant share of variance that can be attributed to respondents' own bespoke neighbourhoods of their closest 20 neighbours. This is especially the case for the indicator of a gentrifying, more affluent neighbourhood. A similar result is obtained for the indicator of an ethnically diverse neighbourhood, although it marginally misses statistical significance at the $5 \%$ level. However, since these results are based on the whole sample, it cannot be ruled out that this association depicts in- 
dividual differences regarding the ethnic background of respondents: Given the levels of ethnic and social segregation in the real world, neighbours in the bespoke neighbourhoods likely have similar individual characteristics. The fact that people born abroad have very similar tastes as those born in Switzerland, especially regarding their evaluation of a housing alternative's social and ethnic composition, nevertheless strengthens an interpretation in line with the theoretical argument. This is also supported by the fact that the heterogeneity among individual respondents was mainly found in their evaluation of the ethnic composition and less in the social composition of a housing alternative's neighbourhood (models 3 and 4 in Table 2). Meanwhile, the pattern is exactly reversed when linking the observed heterogeneity to their experienced neighbourhood conditions.

Summing up, it has been demonstrated how both native Swiss as well as those born abroad show similar residential preferences. However, this first impression, which does not support an ethnic differential in people's housing preferences and thus challenges often-articulated views on the causes of segregation, is somewhat put into perspective when we account for heterogeneous preferences. It has then been shown how people differ in their evaluations of the social and ethnic composition associated with different housing alternatives. Finally, it has been demonstrated how these heterogeneous preferences can partially be linked to people's currently experienced neighbourhood conditions.

\section{Discussion}

Social and ethnic segregation is often conceptualized as a consequence of individual and household residential preferences and housing decisions (Clark and Fossett, 2008; Clark, 1991; Schelling, 1971). Such a view, however, not only neglects structural constraints encountered by different groups when making a housing location decision, but it also tends to treat preferences as exogenous. Emphasizing that residential preferences are formed within the experienced neighbourhood context (Bruch and Feinberg, 2017; Bruch and Mare, 2006), this paper proposes an alternative view. Preferences are conceptualized in a utility maximization perspective in which people evaluate different housing alternatives according to characteristics of the alternatives and person-specific traits (e.g., according to individual ethnic background). Moreover, preferences and tastes are allowed to be influenced by experienced neighbourhood conditions.

Using geocoded data that allows for the construction of small-scale neighbourhoods including the 20 closest door-to-door neighbours in four Swiss cities and a choice experiment, the paper examined to which extent the often documented ethnic residential preferences (Clark, 1991; Fossett, 2006; 
Ibraimovic and Hess, 2018) can be attributed to respondents' own neighbourhoods. Thus, rather than asking to which extent ethnic preferences might explain observed levels of social and ethnic segregation in urban areas, this paper looks at the reverse link of how such segregation patterns are also a potential cause for the heterogeneity in people's residential and housing preferences. To this end, this contribution made use of conditional and mixed logit models as well as a multilevel approach that allows for residential preference heterogeneity to be explained by respondents' own neighbourhood context (Skrondal and Rabe-Hesketh, 2003; Train, 2009).

Results indicate that people born in Switzerland and those with a migration background do not differ systematically in their evaluations of a neighbourhood's social and ethnic composition when making a housing decision in the choice experiment. More importantly, however, the evaluation of both the ethnic as well as the social environment of a housing alternative's neighbourhood is heterogeneous: Although people are generally less likely to prefer an ethnically mixed neighbourhood in the choice experiment, there is a considerable share of respondents who evaluated such neighbourhoods positively. This pattern is especially pronounced in people who were not born in Switzerland. Although this heterogeneity cannot be fully attributed to a (crude) measure of respondents' own ethnic background, a significant share of this taste heterogeneity can be linked to respondents' own bespoke neighbourhoods. At the same time, people's tastes seem not to vary across administrative neighbourhoods. Consequently, this finding stresses small-scale processes at the local level in the explanation of people's heterogeneous responses to the ethnic composition of housing alternatives. Likewise, it also highlights the often documented limitation of using predefined geographical measures such as census blocks or wards to assess neighbourhood conditions (e.g., Coulton et al., 2013; Logan, 2018).

However, there are several limitations to keep in mind. Based on the presented evidence, it cannot be ruled out that a more differentiated measurement of individual ethnic background would reveal significant differences among ethnic groups. Moreover, the bespoke neighbourhoods in this study are very small, comprising only the 20 closest households. While this is due to the sampling strategy of the survey, it also brings the disadvantage of risking a confusion between a contextual effect and unmeasured individual differences. Compared to the larger administrative neighbourhoods, neighbours in these small bespoke neighbourhoods are very similar to each other and one might thus wrongly attribute individual taste heterogeneity that is due to respondents own social and ethnic background to differences across bespoke neighbourhoods. Finally, in the choice experiment respondents were asked to make a recommendation for a friend instead of to chose an adequate housing for themselves. Although this might hinder a straightforward generalization of the documented preferences, this approach also ensures that respondents abstract more from 
their own current housing condition and thus render the estimates more conservative. Similarly, using descriptions of different types of neighbourhood businesses in the choice experiment might not be the best way to measure people's response to the social and ethnic composition of housing alternatives' surroundings. At the same time, alternative measures such as the information about the share of migrant populations (e.g., Ibraimovic and Hess, 2018) might induce social desirability bias. Likewise, people usually do not judge neighbourhoods by means of such statistical information but rather based on daily experiences with people and the infrastructure they encounter in the local context. Nevertheless, a more detailed examination for different ethnic groups and at different geographic levels of (bespoke) neighbourhoods would put the results of this study to the test.

In conclusion, the findings of this paper put the role of the hypothesized and often documented ethnic residential preferences into perspective. As a consequence, explanations of residential segregation that focus solely on the role of people's housing and residential preferences might overestimate their relevance. Rather, both researchers as well as policy makers should investigate the mutual links between experienced neighbourhood conditions, residential mobility decisions, and segregation patterns more closely. Preferences are not exogenous but are formed within the wider socio-spatial context, which is the result of different social processes such as discriminatory practices of landlords, individual mobility decisions, and perceived neighbourhood change (Auspurg et al., 2017; Bruch and Feinberg, 2017; van Ham and Clark, 2009). Future research can thus only benefit from accounting for these mutual dependencies by investigating the heterogeneity in people's residential preferences in more detail. However, the evidence presented in this paper relies on stated rather than revealed preferences (Timmermans et al., 1994). It is likely that situational constraints have a much bigger impact when studying real-world housing decisions. Meanwhile, such constraints might also add their share to the explanation of the documented ethnic residential preferences, because different social and ethnic groups encounter different constraints and opportunities in the local context when making a housing decision. Consequently, such structural influences need to be disentangled from people's preferences. To do so, future research might greatly benefit from combining stated with revealed preferences to circumvent the shortcomings of both approaches.

\section{Notes}

\footnotetext{
${ }^{1}$ The literature suggests to experimentally alter five to nine dimensions (Auspurg and Hinz, 2015).

${ }^{2}$ A significant interaction, for example, of respondents' ethnic background with one of the available alternatives would only imply that this particular group is more likely to choose, say, option B in the choice experiment,
} 
independently of the dimensions describing that alternative.

${ }^{3}$ Likewise, we could also calculate respondents' WTP with regard to the distance to the city centre. Doing so reveals the same pattern (not shown).

${ }^{4}$ Doing so for the conditional and the mixed logit models leads to comparable results as the ones obtained for the native-born subsample, although the effects are generally more significant due to the larger sample size.

\section{References}

Aldén L, Hammarstedt M and Neuman E (2015) Ethnic Segregation, Tipping Behavior, and Native Residential Mobility. International Migration Review 49(1): 36-69. DOI:10.1111/imre.12066. URL http://doi.wiley.com/10.1111/imre.12066.

Auspurg K and Hinz T (2015) Factorial Survey Experiments. Number 175 in Quantitative Applications in the Social Science. Thousand Oaks: Sage.

Auspurg K, Hinz T and Schmid L (2017) Contexts and conditions of ethnic discrimination: Evidence from a field experiment in a German housing market. Journal of Housing Economics 35(1): 26-36. DOI:10.1016/j.jhe.2017.01.003.

Bruch E and Feinberg F (2017) Decision-making processes in social contexts. Annual Review of Sociology 43: 207-227.

Bruch EE and Mare RD (2006) Neighborhood Choice and Neighborhood Change. American Journal of Sociology 112(3): 667-709.

Bruch EE and Mare RD (2012) Methodological Issues in the Analysis of Residential Preferences, Residential Mobility, and Neighborhood Change. Sociological Methodology 42(1): 103-154.

Clark WA and Fossett M (2008) Understanding the social context of the Schelling segregation model. Proceedings of the National Academy of Sciences 105(11): 4109-4114. URL http: //www.pnas.org/content/105/11/4109. short.

Clark WAV (1991) Residential Preferences and Neighborhood Racial Segregation: A Test of the Schelling Segregation Model. Demography 28(1): 1.

Coulton CJ, Jennings MZ and Chan T (2013) How Big is My Neighborhood? Individual and Contextual Effects on Perceptions of Neighborhood Scale. American Journal of Community Psychology 51(1-2): 140-150.

Dieleman FM (2001) Modelling residential mobility; a review of recent trends in research. Journal of housing and the built environment 16(3-4): 249-265. 
Emerson MO, Chai KJ and Yancey G (2001) Does Race Matter in Residential Segregation? Exploring the Preferences of White Americans. American Sociological Review 66(6): 922-935. DOI:10.2307/3088879. URL http://www . jstor . org/stable/3088879? origin=crossref.

Flage A (2018) Ethnic and gender discrimination in the rental housing market: Evidence from a meta-analysis of correspondence tests, 2006-2017. Journal of Housing Economics 41: 251-273. DOI:10.1016/j.jhe.2018.07.003.

Fossett M (2006) Ethnic Preferences, Social Distance Dynamics, and Residential Segregation: Theoretical Explorations Using Simulation Analysis. The Journal of Mathematical Sociology 30(3-4): 185-273. DOI:10.1080/00222500500544052.

Hole AR (2007) A comparison of approaches to estimating confidence intervals for willingness to pay measures. Health Economics 16(8): 827-840. DOI:10.1002/hec.1197.

Ibraimovic T and Hess S (2018) A latent class model of residential choice behaviour and ethnic segregation preferences. Housing Studies 33(4): 544-564. DOI:10.1080/02673037.2017.1373749.

Iceland J and Hernandez E (2017) Understanding trends in concentrated poverty: 1980-2014. Social Science Research 62(1): 75-95. DOI:10.1016/j.ssresearch.2016.09.001.

Ioannides YM (1987) Residential Mobility and Housing Tenure Choice. Regional Science and Urban Economics 17: 265-287.

Ioannides YM (2013) From neighborhoods to nations: The economics of social interactions. Princeton University Press.

Johnson FR, Lancsar E, Marshall D, Kilambi V, Mühlbacher A, Regier DA, Bresnahan BW, Kanninen B and Bridges JF (2013) Constructing Experimental Designs for Discrete-Choice Experiments: Report of the ISPOR Conjoint Analysis Experimental Design Good Research Practices Task Force. Value in Health 16(1): 3-13.

Kuhfeld WF (2010) Marketing research methods in SAS. Cary, NC: SAS Institute Inc. URL http://support. sas. com/techsup/technote/mr2010.pdf.

Lee BA, Oropesa R and Kanan JW (1994) Neighborhood Context and Residential Mobility. Demography 31(2): 249-270.

Logan JR (2018) Relying on the Census in Urban Social Science:. City \& Community 17(3): 540-549. DOI:10.1111/cico.12331. URL http://doi.wiley.com/10.1111/cico.12331. 
Louviere JJ, Street D, Burgess L, Wasi N, Islam T and Marley AA (2008) Modeling the choices of individual decision-makers by combining efficient choice experiment designs with extra preference information. Journal of Choice Modelling 1(1): 128-164. DOI:10.1016/S1755-5345(13)70025-3.

Lévy-Garboua L, Meidinger C and Rapoport B (2006) Chapter 7 The Formation of Social Preferences: Some Lessons from Psychology and Biology. In: Handbook of the Economics of Giving, Altruism and Reciprocity, volume 1. Elsevier. ISBN 978-0-444-50697-9, pp. 545-613. DOI: 10.1016/S1574-0714(06)01007-4.

McFadden D (1974) Conditional Logit Analysis of Qualitative Choice Behavior. In: Zarembka P (ed.) Frontiers in Econometrics. New York: Academic Press, pp. 105-142.

McFadden D (1978) Modeling the choice of residential location. Transportation Research Record 673: $72-77$.

Musterd S (2005) Social and Ethnic Segregation in Europe: Levels, Causes, and Effects. Journal of Urban Affairs 27(3): 331-348.

Rokem J and Vaughan L (2019) Geographies of ethnic segregation in Stockholm: The role of mobility and co-presence in shaping the 'diverse' city. Urban Studies 56(12): 2426-2446. DOI: $10.1177 / 0042098018795561$.

Rossi PH and Shlay AB (1982) Residential mobility and public policy issues:"Why families move" revisited. Journal of Social Issues 38(3): 21-34.

Sampson RJ (2012) Great American City. Chicago and the enduring neighborhood effect. Chicago: Chicago University Press.

Schelling TC (1971) Dynamic Models of Segregation. Journal of Mathematical Sociology 1(1): $143-186$.

Skrondal A and Rabe-Hesketh S (2003) Multilevel logistic regression for polytomous data and rankings. Psychometrika 68(2): 267-287. DOI:10.1007/BF02294801.

Timmermans H, Molin E and Van Noortwijk L (1994) Housing choice processes: Stated versus revealed modelling approaches. Journal of Housing and the Built Environment 9(3): 215-227.

Timmermans H and Noortwijk Lv (1995) Context dependencies in housing choice behavior. Environment and Planning A 27(2): 181-192.

Train KE (2009) Discrete choice methods with simulation. 2 edition. Cambridge University Press. 
van Ham M and Clark WAV (2009) Neighbourhood Mobility in Context: Household Moves and Changing Neighbourhoods in the Netherlands. Environment and Planning 41(6): 1442-1459.

Wang D and $\mathrm{Li} \mathrm{Sm}$ (2006) Socio-economic differentials and stated housing preferences in Guangzhou, China. Habitat International 30(2): 305-326.

Weinberg DH (1979) The Determinants of Intra-Urban Household Mobility. Regional Science and Urban Economics 9: 219-246.

Zangger C and Becker R (2019) Experiments in the sociology of education: causal inference and estimating causal effects in sociological research on education. In: Becker R (ed.) Research Handbook on the Sociology of Education, Research Handbooks in Sociology. Cheltenham: Edward Elgar, pp. 153-171. 